\title{
Case Report: Severe brain hypoxic damages after acute methanol poisoning
}

\author{
Ameni Abidi ${ }^{1}$, Asma Souid ${ }^{2}$, Khalaf Ben Abdallah ${ }^{1}$, Nadia Hammami ${ }^{2}$, Selima Siala ${ }^{2}$, \\ Nozha Brahmi ${ }^{1}$, and Mohamed Ben Hamouda ${ }^{2}$ \\ ${ }^{1}$ Department of Intensive care and Toxicology, Centre d'assistance médicale d'urgence \\ CAMU \\ ${ }^{2}$ Mongi-Ben Hamida National Institute of Neurology Department of Neuroradiology
}

February 17, 2022

\begin{abstract}
Methanol poisoning is a challenging clinical situation with irreversible neurologic complication mainly encountered in developed countries. We report a case of a 50-year-old patient who presented with methanol poisoning, symptomatic of respiratory and neurologic failure. In this context, cerebral magnetic resonance imaging concluded entangled injury mechanisms leading to neurologic failure.
\end{abstract}

Case Report: Severe brain hypoxic damages after acute methanol poisoning Abidi $\mathrm{A}^{1}$, Souid $\mathrm{A}^{2}$, B Abdallah $\mathrm{K}^{1}$, Hammami $\mathrm{N}^{2}$, Siala $\mathrm{S}^{2}$, Brahmi $\mathrm{N}^{1}$, B Hamouda $\mathrm{M}^{2}$

${ }^{1}$ Department of Intensive care and Toxicology, Centre d'assistance médicale d'urgence CAMU, Tunis 1089, Tunisia

${ }^{2}$ Department of Neuroradiology, Mongi Ben Hamida National Institute of Neurology, Tunis 1007, Tunisia

List of Authors:

Abidi Ameni, MD: drabidiameni@gmail.com

Souid Asma, MD: asmasouid5@gmail.com

Ben Abdallah Khalaf, MD:khalaf.benabdallah14@gmail.com

Hammami Nadia, MD: nadiahamaied@hotmail.com

Siala Salima, MD: sialaselima@gmail.com

Brahmi Nozha, MD: nzh.brahmi@gmail.com

Ben Hamouda Mohamed, MD: benhamouda.mohamed93@gmail.com

Corresponding Author:

Khalaf Ben Abdallah

khalaf.benabdallah14@gmail.com, Phone number: +21651838246

Address: 15 Road 6828 Omran Sup 1091 Tunis

Keywords 
case report, methanol poisoning, hypoxia, haemorrhagic necrosis, white matter demyelination, magnetic resonance imaging

Consent

Written informed consent for publication of their clinical details and clinical images was obtained from the patient's family.

\section{Abstract:}

Methanol poisoning is a challenging clinical situation with irreversible neurologic complication mainly encountered in developed countries. We report a case of a 50-year-old patient who presented with methanol poisoning, symptomatic of respiratory and neurologic failure. In this context, cerebral magnetic resonance imaging concluded entangled injury mechanisms leading to neurologic failure.

\section{Key message:}

In methanol poisoning, cerebral magnetic resonance imaging is essential in identifiying neurologic injury mechanisms. Secondary hypoxia in acute methanol poisoning can worsen the final outcome.

\section{Introduction:}

Hypoxic-ischemic brain injury is common after cardiac arrest. Severe brain damage can be observed after prolonged brain hypoxia following some intoxications, with certain specificities for methanol [1]. Imaging and mainly magnetic resonance imaging (MRI) is done to assess the amount of central nervous system damage, the prognosis as well as the outcome. We present a case of severe putaminal haemorrhagic necrosis and diffuse white matter damage due to both methanol poisoning and severe hypoxia with a poor prognosis.

Case report:

We present a case of a 50-year-old male patient, with a 20-year history of addiction to alcohol as well as recent ingestion of an amount of cologne water instead of alcohol for economic reasons. He presented in our Tunis Centre d'assistance médicale d'urgence's emergency department, in April 2020, with blurred vision and diplopia 21 hours after the ingestion of one liter of an unusual, commercialized liquor containing $70 \%$ formalin. A few hours later, he lapsed into a coma and had respiratory distress with $\mathrm{SpO}_{2}$ of $77 \%$. Mechanical ventilation was required for advanced airway management and he was transferred into our ICU. Investigations showed severe metabolic acidosis: $\mathrm{pH}=6.87, \mathrm{PaCO}_{2}=25 \mathrm{mmHg}, \mathrm{HCO}_{3^{-}}=4.6 \mathrm{mmol} / \mathrm{L}$ and elevated anion gap of $27 \mathrm{mEq} / \mathrm{L}$ with hyperlactatemia of $5 \mathrm{mmol} / \mathrm{L}$. Toxicology and drug screen in blood samples revealed high methanol levels of $6.23 \mathrm{~g} / \mathrm{L}$. The patient was given $40 \%$ ethanol as a bolus of $0.6 \mathrm{~g} / \mathrm{kg}$ and a maintenance dose of $100 \mathrm{mg} / \mathrm{kg} / \mathrm{h}$ in association to 42sessions of six hours each, blood pump speed of $200 \mathrm{~mL} / \mathrm{min}$, adjuvant treatment with intravenous folic acid $200 \mathrm{mg}$ daily and intravenous Vitamin B1 300 mg daily.

Brain MRI performed on the seventh day showed bilateral symmetrical putaminal signal anomalies (Figure 1) consisting of heterogeneous hyperintensities in T1, T2 and Fluid-attenuated inversion recovery (FLAIR) sequences containing low signal regions on $\mathrm{T} 2 *$ weighted gradient echo and low apparent diffusion coefficient (ADC) value regions on diffusion weighted images. These findings represent the putaminal hemorrhagic necrosis. MRI showed also diffuse bilateral symmetrical extensive white matter abnormal signal intensity showing a T2/FLAIR hyperintensity, a T1 hypointensity with low ADC values on diffusion weighted images and respecting sub-cortical U-fibers (Figure 2). There was no hemorrhagic stigma in the white matter. There was no restricted diffusion in the retrobulbar segment of the optic nerves. Written informed consent for publication of their clinical details and clinical images was obtained from the patient's family.

The electroencephalogram done on day 26 showed a slow disorganized, non-reactive pattern, indicating diffuse brain suffering. The patient did not demonstrate neurological recovery. On day 32, refractory hypoxia under mechanical ventilation leaded to his death.

Discussion: 
Putaminal hemorrhagic necrosis can represent an early consequence of cerebral hypoxia in general but it is most often seen after carbon monoxide intoxication. However, this finding can also happen in isolated methanol intoxication [1]. In our case, we cannot differentiate whether putaminal lesions were due to hypoxemia or specific methanol toxicity. In fact, formic acid formation secondary to methanol poisoning, can inhibit cytochrome oxidase activity in the mitochondria, leading to histotoxic hypoxia with predilection for the brain and the visual pathway [2,3]. Reports have described an association between extensive white matter demyelination and basal ganglionic damage after hypoxia [4]. This damage is explained in our case probably by the delay of resuscitation and therefore brain hypoxia. In fact, hypoxia causes necrosis and edema in different regions in the brain. Cerebral cortex, hippocampus, cerebral cortex as well as caudate nucleus, putamen and globus pallidus are considered as vulnerable zones in the brain [5]. Putamen is prone to develop injuries because of its high metabolic demand and its crucial place in vascular perfusion's boundary zones [6].

The extension of these lesions depends on hemodynamic and metabolic patient characteristics but mostly the duration of hypoxia. Individuals older than 30 years like our patient are at greater risk of delayed demyelination [7].

Evolution varies from recovery to death. In our case the patient did not demonstrate neurological recovery.

As for other methanol intoxication MRI findings, optic nerve damage can be assessed by enhanced T1 fatsaturated (FatSat) images and diffusion-weighted images showing a bilateral mild contrast enhancement and restricted diffusion in the retrobulbar segment of the optic nerves [8]. In our case, there was no restricted diffusion in the optic nerves.

Our case is interesting because it represents severe methanol intoxication associated with a severe hypoxic ischemic brain injury. MRI was crucial for the diagnosis of this damage. We attest the lack of a $2^{\text {nd }}$ control MRI after antidote therapy which could have given us a thorough understanding of the injury evolution in our patient.

\section{Conclusion}

In severe methanol intoxication, both brain prolonged hypoxia and the intoxication itself can lead to severe putaminal hemorrhagic necrosis. Diffuse white matter demyelination is, however, due the delay of resuscitation and therefore brain hypoxia; it serves to make the patient's prognosis poorer and worsen his outcome.

Data availability

All data underlying the results are available as part of the article and no additional source data are required.

\section{Consent}

Written informed consent for publication of their clinical details and clinical images was obtained from the patient's family.

\section{Competing interests}

No competing interests were disclosed.

\section{Grant information}

The authors declared that no grants were involved in supporting this work.

Author contributions:All authors were involved in the researching, writing and editing of the manuscript.

\section{References}

1. Rubinstein D, Escott E, Kelly JP. Methanol intoxication with putaminal and white matter necrosis: MR and CT findings. AJNR Am J Neuroradiol. 1995; 16(7): 1492-4. 
2. Hantson PE. Acute methanol intoxication: physiopathology, prognosis and treatment. Bull MemAcad R Med Belg. 2006; 161: 425-34. discussion 434-436.

3. Brahmi N, Blel Y, Abidi N, Kouraichi N, Thabet H, Hedhili A, Amamou M. Methanol poisoning in Tunisia: report of 16 cases. ClinToxicol (Phila). 2007; 45: 717-720.

4. Lou M, Jing C-H, Selim MH, Caplan LR, Ding M-P. Delayed substantia nigra damage and leukoencephalopathy after hypoxic-ischemic injury. J Neurol Sci. 2009; 277(1-2): 147-9.

5. Caplan LR. Cardiac arrest and other hypoxic ischemic insults. In: Caplan LR, Hurst JW, Chimowitz M, editors. Clinical neurocardiology. New York: Marcel Dekkar. 1999; pp 1-39.

6. Hsu HH, Chen CY, Chen FH, Lee CC, Chou TY, Zimmerman RA. Optic atrophy and cerebral infarcts caused by methanol intoxication: MRI. Neuroradiology. 1997; 39(3): 192-4.

7. Gottfried JA, Mayer SA, Shungu DC, Chang Y, Duyn JH. Delayed posthypoxic demyelination. Association with arylsulfatase A deficiency and lactic acidosis on proton MR spectroscopy. Neurology. 1997; 49(5): $1400-4$.

8. Tanrivermis Sayit A, Aslan K, Elmali M, Gungor I. Methanol-induced toxic optic neuropathy with diffusion weighted MRI findings. Cutan Ocul Toxicol. 2016; 35(4): 337-40.

Figure legends:

Figure 1: Axial brain magnetic resonance images showing putaminal hemorragic necrosis (arrows):

A: FLAIR sequence showing bilateral heterogeneous putaminal hyperintensities surrounded by hypointense borders. B: T1 weighted image: high signal on putaminal affected area representing the hemorrhagic nature. C: Putaminal low signal regions on the $\mathrm{T}^{*}$ sequence related to a hemosiderine component. We note also the diffuse subcortical white matter demyelination $(\mathrm{A}, \mathrm{B}, \mathrm{C})$

Figure 2: Axial brain magnetic resonance images showing diffuse subcortical white matter demyelination:

$\mathrm{A}+\mathrm{B}$ : T2 weighted image (A) and FLAIR sequence (B) showing bilateral diffuse subcortical white matter high signal, sparing subcortical U fibers and deep periventricular white matter.

C: Hypointense subcortical white matter on T1 weighted image. D + E: This extensive demyelination shows high signal intensity on B1000 diffusion weighted image (D) with low apparent diffusion coefficient (ADC) values (arrows).

\section{Hosted file}

Figures-.docx available at https://authorea.com/users/461094/articles/556865-case-reportsevere-brain-hypoxic-damages-after-acute-methanol-poisoning 